\title{
Management of fetal tachyarrhythmia based on superior vena cava/aorta Doppler flow recordings
}

\author{
J-C Fouron, A Fournier, F Proulx, J Lamarche, J L Bigras, C Boutin, M Brassard, \\ S Gamache
}

See end of article for authors' affiliations

\section{Correspondence to:}

Dr J-C Fouron, Cardiology

Division, Sainte-Justine

Hospital, 3175, Côte

Ste-Catherine, Montreal,

Quebec H3T 1C5,

Canada;

fouron@sympatico.ca

Accepted 21 May 2003

\begin{abstract}
Objective: To evaluate a management protocol of fetal supraventricular tachycardia (SVT) based on prior identification of the underlying mechanism.

Design and setting: Prospective study in a mother-child tertiary university centre.

Patients: During a consecutive 36 month period, 18 fetuses with sustained SVT underwent a superior vena cava/ascending aorta (SVC/AA) Doppler investigation in an attempt to determine the atrioventricular $(\mathrm{AV})$ relation and to treat the arrhythmia according to a pre-established management protocol.

Main outcome measure: Rate of conversion to sinus rhythm.

Results: Seven fetuses had short ventriculoatrial tachycardia, five of these with a 1:1 AV conduction suggesting re-entrant tachycardia. The first choice drug was digoxin and all were converted. One fetus had AV dissociation leading to the diagnosis of junctional ectopic tachycardia, which was resistant to digoxin and sotalol; amiodarone achieved postnatal conversion. One fetus had SVT and first or second AV block; the diagnosis was atrial ectopic tachycardia (AET), which responded to sotalol given as a drug of first choice. Seven fetuses had long ventriculoatrial tachycardia: one with sinus tachycardia (no treatment), one with permanent junctional reciprocating tachycardia (PJRT), and three with AET. The first choice drug was sotalol and all were converted. One AET was classified postnatally as PJRT. Six fetuses had intra-atrial re-entrant tachycardia: five with 2:1 AV conduction and one with variable block. The first choice drug was digoxin. Conversion was achieved in all but one, who died after birth from advanced cardiomyopathy.

Conclusion: The electrophysiological mechanisms of fetal SVT can be clarified with SVC/AA Doppler. The proposed management protocol has so far yielded a good rate of conversion to sinus rhythm.
\end{abstract}

dia n extrauterine life, narrow QRS supraventricular tachycardia (SVT) is conveniently classified according to the relation of P waves to QRS complexes. ${ }^{1}$ During intrauterine life, such a classification has been hampered by the very low voltage of $\mathrm{P}$ waves on transmaternal fetal ECGs. Consequently, digoxin is advocated as the drug of first choice for all cases of fetal SVT, without discrimination. In an attempt to investigate the potential utility of ultrasound approaches in the differentiation of fetal tachycardia, we previously found that fetal M mode echocardiograms may help in identifying fetuses with short ventriculoatrial (VA) and long VA tachycardia. ${ }^{2}$ However, $\mathrm{M}$ mode recording of the fetal heart has its limitations, the two most significant being, firstly, the loss of clear markers of atrial and ventricular contractions in hydropic fetuses with hypocontractile myocardium and, secondly, the poor picture resolution related to factors such as maternal obesity, fetal position, or polyhydramnios. Simultaneous Doppler recording of flow velocities in the superior vena cava and ascending aorta (SVC/AA) has been shown to be another ultrasound approach more accurate than $M$ mode for atrioventricular $(\mathrm{AV})$ and VA interval measurements. ${ }^{3}$ For this reason, a prospective investigative protocol has been established in our unit in which all fetuses referred for tachyarrhythmia undergo SVC/AA Doppler recordings in an attempt to identify the underlying mechanism before initiating any drug treatment.

In this report, we describe our management protocol specifically designed to address the mechanism of the arrhythmia and to present a preliminary assessment of the impact of this protocol on the outcome of affected fetuses.

\section{METHODS}

During a consecutive 36 month period starting in 1999, 491 fetuses were sent to our unit for investigation of arrhythmia.
From this group, 18 had sustained tachycardia with heart rate above 180 beats/min. The remaining fetuses had isolated premature atrial contractions, rarely sustained bradycardia, or transient tachycardia. The technique used to obtain an SVC/AA Doppler recording, as fig l shows, has been described previously. ${ }^{3}$ Tachyarrhythmia was defined as sustained when present during more than $50 \%$ of the 30-45 minute period that was necessary for a complete echocardiographic investigation. Only sustained tachyarrhythmia was included in this study. A fetus was considered hydropic when subcutaneous oedema was associated with fluid collection in at least one location, such as the pleural, pericardial, or peritoneal cavity.

Figure 2 summarises our treatment protocol. For all tachyarrhythmias that did not convert to sinus rhythm after five days of first choice drug treatment, the decision whether to continue the same medication or to add another drug was based both on the fetal condition (presence or absence of hydrops) and the gestational age (before or after 35 weeks of pregnancy). Since long VA tachycardia is known to be difficult to convert, fetal delivery would be considered even before 35 weeks of pregnancy if, after 5-7 days of the two drug regimens planned in the protocol, no change was observed in the tachycardia.

Maternal ECG, blood potassium, and calcium were all assessed on the first day of treatment. The ECG was repeated twice a week to monitor individual susceptibility to digoxin or

Abbreviations: $A A$, ascending aorta; $A E T$, atrial ectopic tachycardia; $\mathrm{AV}$, atrioventricular; PJRT, permanent junctional reciprocating tachycardia; SVC, superior vena cava; SVT, supraventricular tachycardia; VA, ventriculoatrial 


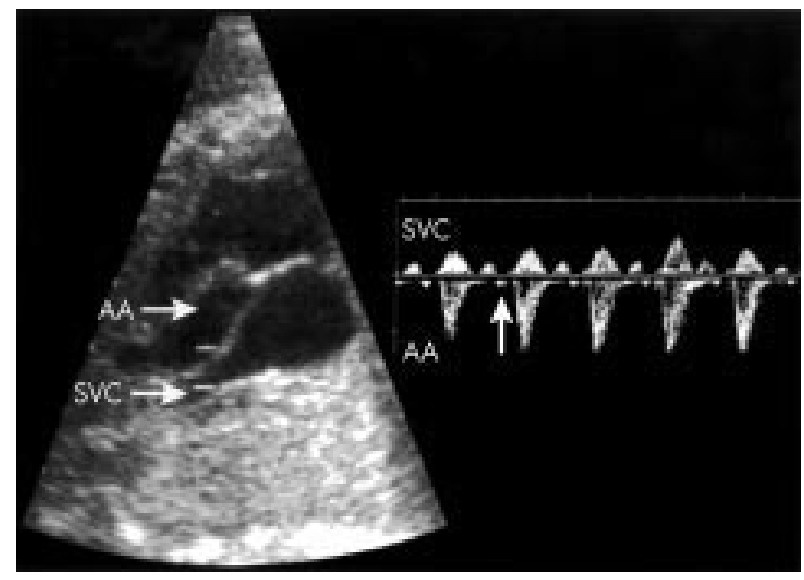

Figure 1 Left: Two dimensional imaging of a segment of the ascending aorta (AA) adjacent to the superior vena cava (SVC) draining into the right atrium. The widened Doppler sampling volume encompasses the aorta and SVC. Right: Doppler tracing obtained from the same patient. Aortic ejection waves are recorded below the zero velocity line. Anterograde venous flow in the SVC going in the opposite direction is above the same line. Each aortic ejection wave is preceded by a small venous retrograde wave caused by right atrial contraction (arrow).

sotalol. Maternal digoxinaemia was maintained between $1-2 \mu \mathrm{g} / 100 \mathrm{ml}$. Fetal intravenous administration of digoxin was given for short VA tachycardia in advanced hydrops through cordocentesis at a dose of $0.1 \mathrm{mg} / \mathrm{kg}$, followed by adenosine at an initial dose of $50 \mu \mathrm{g} / \mathrm{kg}$ with an increment of $50 \mu \mathrm{g} / \mathrm{kg}$ every 10 minutes for a maximum of $250 \mu \mathrm{g} / \mathrm{kg}$. Maternal blood pressure and heart rate were monitored every six hours throughout the time of hospitalisation needed for initiation of sotalol administration. As long as tachycardia was present, fetal surveillance included heart rate monitoring every four hours every day, associated with echocardiograms and non-stress tests, done on alternate days. Successful treatment was defined as full or at least $>50 \%$ conversion to sinus rhythm after five days of treatment. After conversion to sinus rhythm, the fetus and mother were assessed once a week, alternatively at the high risk pregnancy clinic and at our fetal cardiology unit. After birth, an effort was made to identify the type of arrhythmia when present and thus ascertain the precision of the prenatal diagnosis. If the newborn was in sinus rhythm at the time of delivery, the medication was withheld and reinstated only in case of recurrence.

\section{RESULTS}

The VA relation was established in all 18 fetuses with the SVC/AA approach. Except for one fetus with probable rhabdomyoma, no major morphological cardiac anomaly was found. Tables 1 and 2 summarise the distribution of the fetuses and newborns according to the mechanism of their arrhythmia, management, and outcome.

\section{Short VA tachycardia}

Prenatal data

Seven fetuses had VA intervals shorter than AV (table 1). Five fetuses of this group had a distinctive Doppler pattern characterised by 1:1 AV conduction and a tall A wave (cannon wave) superimposed on the aortic ejection wave, allowing reliable measurement of the VA and AV intervals (fig 3). The duration of the VA interval of the five fetuses averaged $83 \mathrm{~ms}$ (range 64-100 ms). Sudden arrest and onset of the tachyarrhythmia related to atrial ectopy was also documented clearly by SVC/AA Doppler, as fig 3 illustrates. On the basis of these elements, all five fetuses were considered to have AV re-entrant tachycardia through a fast conducting AV accessory pathway. They were treated successfully with digoxin. Fetus 1 received sotalol because of false interpretation of the tracing while improving after three days of digoxin.

In case 6, the presence of AV dissociation excluded the diagnosis of $\mathrm{AV}$ re-entrant tachycardia; the ventricles were

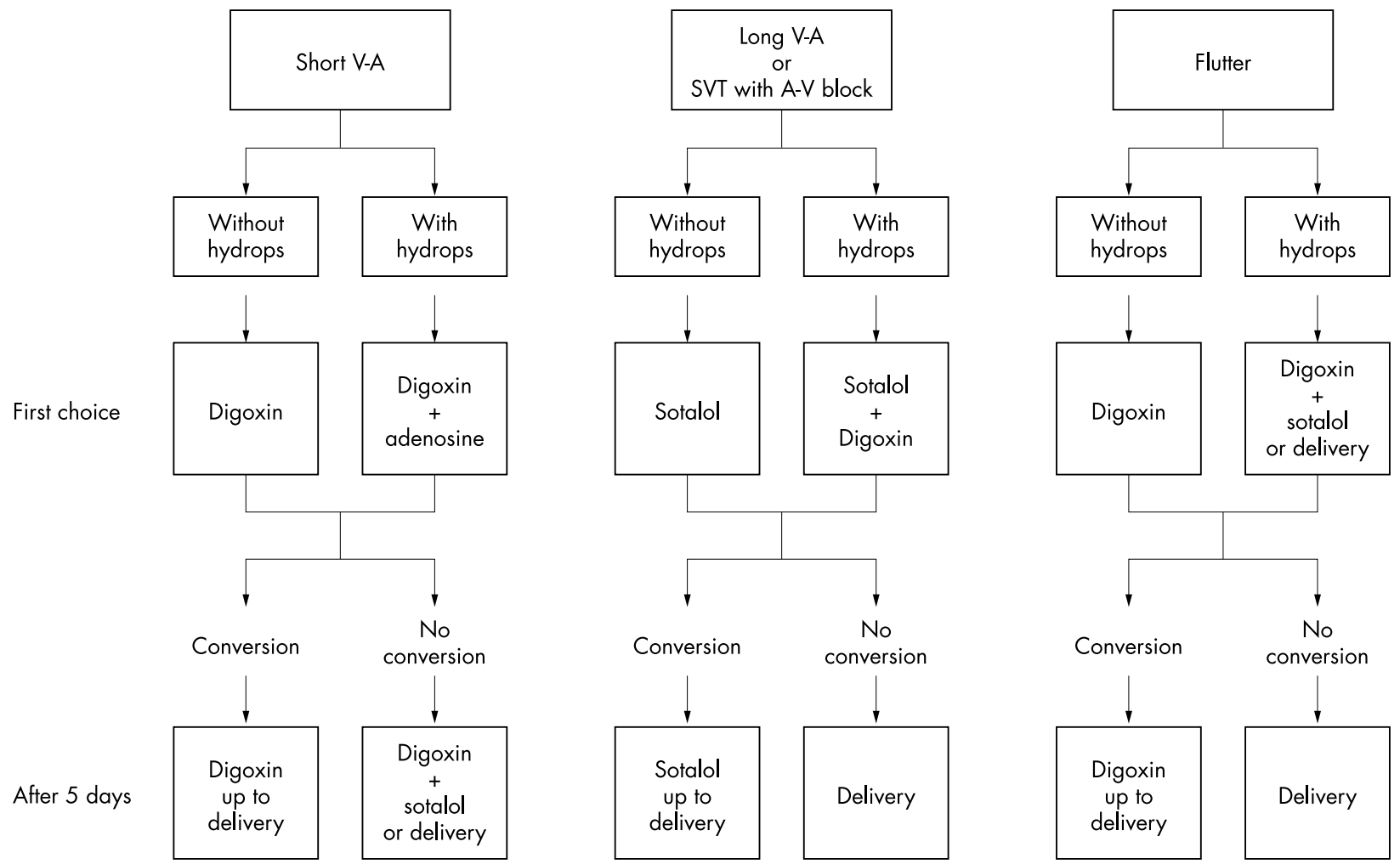

Figure 2 Treatment protocol for fetal tachyarrhythmia. AV, atrioventricular; SVT, supraventricular tachycardia; VA, ventriculoatrial. 
Table 1 Prenatal diagnosis, management, and outcome of 18 fetuses with tachyarrhythmia

\begin{tabular}{|c|c|c|c|c|c|c|c|c|c|c|}
\hline \multirow[b]{2}{*}{ Fetus } & \multirow{2}{*}{$\begin{array}{l}\text { Gestational age } \\
\text { at diagnosis } \\
\text { (weeks) }\end{array}$} & \multirow[b]{2}{*}{$\begin{array}{l}\text { Heart rate } \\
\text { (beats/min) }\end{array}$} & \multirow[b]{2}{*}{ Hydrops } & \multicolumn{2}{|c|}{ Time interval (ms) } & \multirow[b]{2}{*}{ Diagnosis } & \multicolumn{2}{|c|}{ Treatment } & \multicolumn{2}{|l|}{ Delivery } \\
\hline & & & & AV & VA & & $\begin{array}{l}\text { First } \\
\text { choice }\end{array}$ & $\begin{array}{l}\text { Second } \\
\text { choice }\end{array}$ & $\begin{array}{l}\text { Gestational } \\
\text { age (weeks) }\end{array}$ & Mode \\
\hline \multicolumn{11}{|c|}{ Short VA supraventricular tachycardia } \\
\hline 1 & 22 & 240 & None & 168 & 80 & AVRT & Digoxin & Sotalol & 39 & Vaginal \\
\hline 2 & 29 & 240 & None & 150 & 92 & AVRT & Digoxin & & 37 & Vaginal \\
\hline 3 & 33 & 240 & None & 190 & 100 & AVRT & Digoxin & & 40 & Vaginal \\
\hline 4 & 33 & 288 & Severe & 152 & 80 & AVRT & Digoxin & & 34 & CS \\
\hline 5 & 35 & 230 & None & 188 & 64 & AVRT & Digoxin & & 37 & Vaginal \\
\hline 6 & 34 & Atrial 130 Ventricular 200 & Mild & 0 & 0 & JET & Digoxin & Sotalol & 36 & $\mathrm{CS}$ \\
\hline 7 & 31 & 220 & None & 130 & 105 & AET + block & Sotalol & & 38 & Vaginal \\
\hline \multicolumn{11}{|c|}{ Long VA supraventricular tachycardia } \\
\hline 8 & 35 & 200 & None & 128 & 184 & Sinusal & & & 37 & Vaginal \\
\hline 9 & 32 & 210 & None & 139 & 162 & PJRT & Sotalol & Digoxin & 37 & CS \\
\hline 10 & 33 & 190 & None & 152 & 200 & AET & Sotalol & Digoxin & 40 & Vaginal \\
\hline 11 & 37 & 220 & Mild & 88 & 153 & AET & & & 37 & $\mathrm{CS}$ \\
\hline 12 & 35 & 210 & Mild & 117 & 158 & AET & Sotalol & Digoxin & 40 & Vaginal \\
\hline \multicolumn{11}{|c|}{ Intra-atrial re-entrant tachycardia } \\
\hline 13 & 36 & Atrial 500 Ventricular 250 & Severe & & & $F(1: 1,2: 1)$ & Digoxin & & 36 & CS \\
\hline 14 & 27 & Atrial 350 Ventricular 180 & None & & & $F(2: 1)$ & Digoxin & & 40 & CS \\
\hline 15 & 31 & 216 & Severe & & & $F(2: 1)$ & Digoxin & Sotalol & 32 & CS \\
\hline 16 & 33 & Atrial 480 Ventricular 240 & None & & & $F(2: 1)$ & Digoxin & Sotalol & 38 & Vaginal \\
\hline 17 & 31 & Atrial 300 Ventricular 180 & None & & & $F(2: 1)$ & Digoxin & & 40 & Vaginal \\
\hline 18 & 31 & Atrial 0 Ventricular 0 & None & & & $F(2: 1)$ & Digoxin & & 40 & Vaginal \\
\hline
\end{tabular}

$\mathrm{AET}$, atrial ectopic tachycardia; AV, atrioventricular interval; AVRT, atrioventricular re-entrant tachycardia; $C S$, caesarean section; $F$, flutter; HR, heart rate; JET, junctional ectopic tachycardia; PJRT, permanent junctional reciprocating tachycardia; VA, ventriculoatrial interval.

contracting at a rate of $200-210$ beats/min and the atria at a lower, regular pace of $120-140$ beats/min (fig 4). In a brief period of 1:1 conduction, atrial contractions occurred a few milliseconds after or, in some complexes, simultaneously with ventricular contractions (fig 4), making ventricular tachycardia unlikely and leading to the final diagnosis of congenital junctional ectopic tachycardia. This fetus did not respond to the two drugs (digoxin and sotalol) used in our protocol and had to be delivered at 36 weeks in mild hydrops. Fetus 7 presented at 31 weeks with a fixed atrial rate of 220 beats/min but with an AV relation alternating between $1: 1$ and 2:1 conduction, excluding a re-entry through an AV accessory pathway. When 1:1 conduction was present, the AV interval was so prolonged that the VA interval appeared to be shorter than the AV (fig 5). This pattern suggested the presence of an atrial ectopic tachycardia (AET) causing AV node exhaustion leading to either a first or second degree block. In accordance with our protocol, the arrhythmia was successfully treated with sotalol.

\section{Postnatal data}

Of the five newborns with a prenatal diagnosis of AV re-entrant tachycardia, two (cases 4 and 5) had a Wolff-Parkinson-White syndrome and were administered sotalol (table 2). The diagnosis of junctional ectopic tachycardia was confirmed in the sixth neonate (case 6), who responded to amiodarone; the medication was stopped after eight months, as ECG and Holter recordings became normal when the child was 6 months old.

\section{Long VA tachycardia}

\section{Prenatal data}

Five fetuses had sustained tachycardia with VA greater than $\mathrm{AV}$ (table 1). All five fetuses clearly had A waves of normal

Table 2 Postnatal management and outcome of 18 newborns with tachyarrhythmia

\begin{tabular}{|c|c|c|c|c|c|}
\hline Neonate & Weight (kg) & ECG & Treatment & Outcome & Follow up (months) \\
\hline \multicolumn{6}{|c|}{ Short VA supraventricular tachycardia } \\
\hline 1 & 2.975 & Normal & None & No arrhythmia & 28 \\
\hline 2 & 3.275 & Normal & None & No arrhythmia & 29 \\
\hline 3 & 3.555 & Normal & None & No arrhythmia & 18 \\
\hline 4 & 2.485 & WPW & Sotalol during 1 month & No relapse & 13 \\
\hline 5 & 3.785 & WPW & Sotalol & SVT once & 6 \\
\hline 6 & 3.070 & JET & Digoxin, sotalol, amiodarone 8 months & Normal ECG at 6 months & 24 \\
\hline 7 & 2.790 & Normal & None & No arrhythmia & 6 \\
\hline \multicolumn{6}{|c|}{ Long VA supraventricular tachycardia } \\
\hline 8 & 3.100 & Sinus tachycardia & None & No arrhythmia & 28 \\
\hline 9 & 3.530 & PJRT on Holter & Sotalol & No relapse & 8 \\
\hline 10 & 3.065 & PAC, PJRT at 2 weeks & Sotalol 11 months & No relapse & 24 \\
\hline 11 & 3.030 & PAC, AET & Digoxin, sotalol, flecainide inefficacious & $\begin{array}{l}\text { Pacemaker for symptomatic } \\
\text { bradycardia }\end{array}$ & 29 \\
\hline 12 & 3.210 & $\mathrm{AET}$ at 2 weeks & Sotalol & No relapse & 12 \\
\hline \multicolumn{6}{|c|}{ Intra-atrial re-entrant tachycardia } \\
\hline 13 & 3.415 & Flutter & Digoxin, sotalol & Conversion later & 14 \\
\hline 14 & 4.100 & Normal & None & No arrhythmia & 9 \\
\hline 15 & 2.075 & $\begin{array}{l}\text { SVT with } 1: 1 \text { conduction, VA } 100 \mathrm{ms,} \\
\text { WPW in rare sinus beats }\end{array}$ & $\begin{array}{l}\text { Cardioversion with adenosine, digoxin, } \\
\text { amiodarone, amines }\end{array}$ & $\begin{array}{l}\text { Death at } 1 \text { day, sustained } \\
\text { tachycardia }\end{array}$ & \\
\hline 16 & 3.720 & Normal & None & No arrhythmia & 12 \\
\hline 17 & 3.710 & Normal & None & No arrhythmia & 5 \\
\hline 18 & 3.285 & Normal & None & No arrhythmia & 9 \\
\hline
\end{tabular}



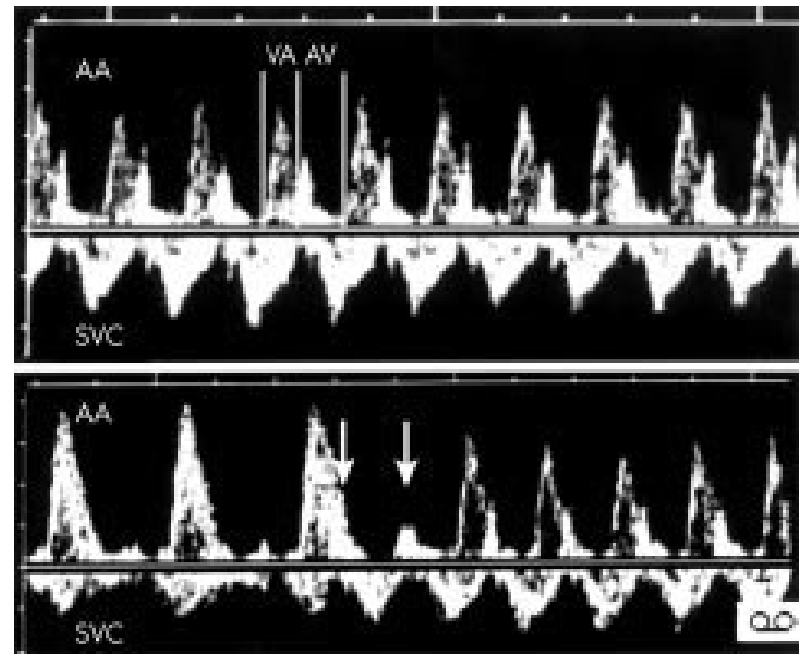

Figure 3 Top: SVC/AA recording in a case (fetus 2) of atrioventricular re-entrant tachycardia. Bottom: In the same patient, sudden onset of the tachycardia triggered by supraventricular couplets (arrows) is shown.

amplitude between the aortic wave forms (fig 6). Fetus 8, first seen at 35 weeks of pregnancy, had a heart rate between 190200 beats/min, rising with fetal activity and slowing with rest. Maternal, clinical, and laboratory investigations including thyroid function were normal. The prenatal diagnosis was sinus tachycardia, probably caused by increased automaticity of the sinus node. No medication was administered.

In another fetus of this group (case 9) there was evidence of sudden arrest and onset of a 1:1 tachyarrhythmia (fig 6
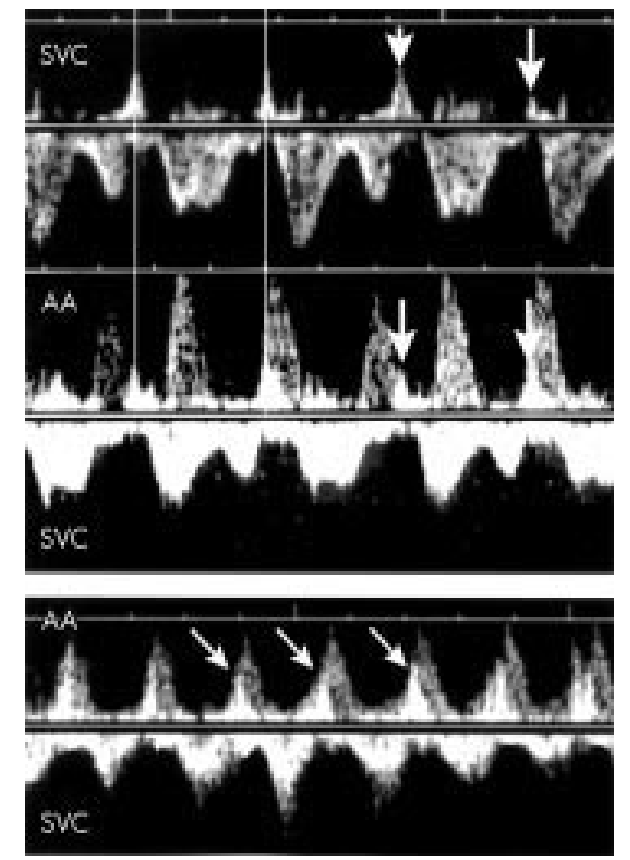

Figure 4 Top: Doppler flow velocity recordings in the SVC of fetus 5 showing an atrial rate of 130 beats/min (arrows). Middle: The SVC/AA recording shows that the ventricles are contracting at the faster rate of 200 beats/min leading to the diagnosis of ventricular tachycardia with AV dissociation. The two vertical lines join the venous " $a$ " waves and illustrate that the atrial rate of contraction is the same on both tracings. Bottom: During a brief period of 1:1 conduction in the same patient, the SVC/AA Doppler tracing shows atrial contractions (arrows) occurring simultaneously or a few milliseconds after ventricular contractions, confirming the diagnosis of junctional ectopic tachycardia.

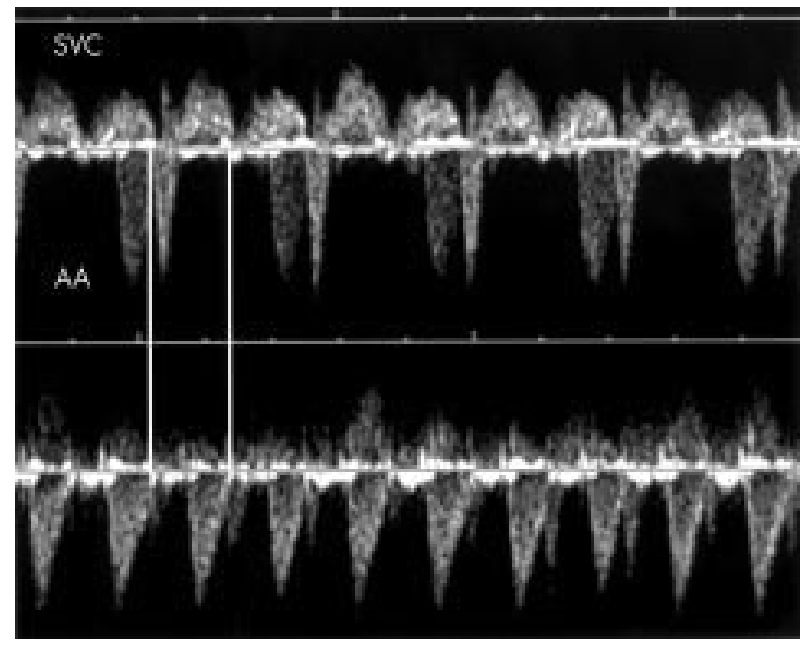

Figure 5 Pulsed Doppler tracing obtained from fetus 6. Top: A fixed atrial rate at 220 beats $/ \mathrm{min}$ is associated with a 2:1 atrioventricular conduction. Bottom: The atrial rate is the same (vertical lines) but a 1:1 conduction is observed. The AV time interval is then so prolonged that the VA appears shorter than the AV. In 1:1 conduction, the $A$ waves are prominent because atrial contractions occur while the tricuspid valve is closed. In the presence of a 2:1 relation (top), the blocked $A$ waves are even taller due to greater blood volume accumulated in the right atrium.

bottom) triggered by extrasystoles, suggesting the possibility of re-entry through a retrograde pathway with slow conduction properties. The diagnosis of permanent junctional reciprocating tachycardia (PJRT) was then considered. According to our protocol, sotalol was the antiarrhythmic agent of first choice but because of mild ascites, digoxin was added to the medication. At day 11 after treatment, digoxin was stopped.

Three fetuses from this group $(10,11$, and 12) had a sustained long VA tachycardia. AET was suspected. Both sotalol and digoxin were administered except to fetus 11 . Conversion to sinus rhythm was documented seven days after initiation of treatment and digoxin was then stopped. In case 11 , echocardiographic study showed a $1.35 \times 1.01 \mathrm{~cm}$ mass in the
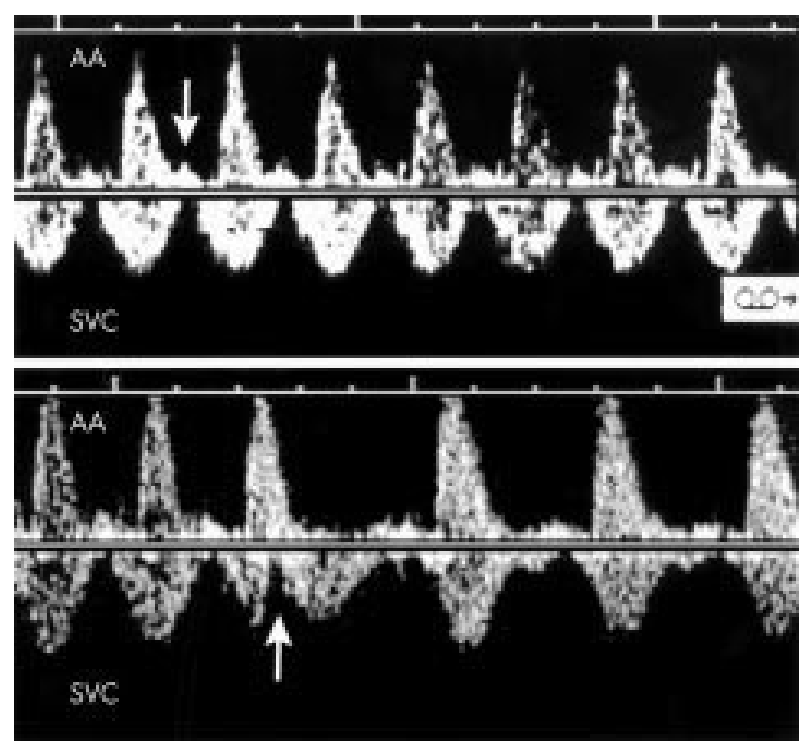

Figure 6 Top: Example of a long VA tachycardia. The A waves are of normal amplitudes (arrow). Bottom: In the same patient, sudden arrest of the tachycardia is observed following premature atrial contraction (arrow), suggesting the possibility of a permanent junctional reciprocating tachycardia. 


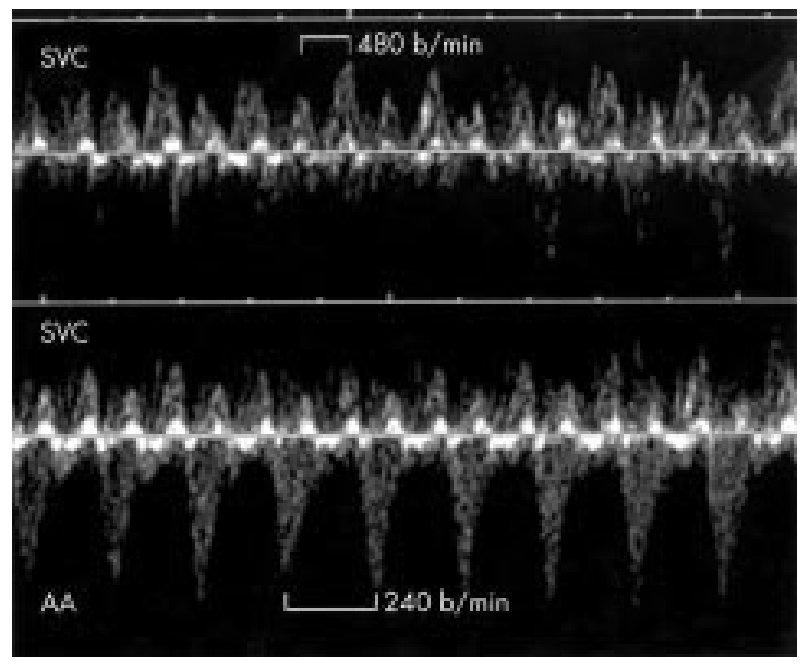

Figure 7 Top: Doppler flow velocity waveforms in the SVC of fetus 15 showing atrial flutter waves at the rate of 480 beats/min. Bottom: The SVC/AA recording in the same patient shows a $2: 1$ relation between the flutter waves and ventricular contractions.

posterior wall of the right atrium and three smaller tumours in the septum and left ventricular free wall. Considering the state of fetal maturation, it was decided not to start prenatal treatment and the fetus was delivered shortly after the diagnosis

\section{Postnatal data}

Sinus tachycardia was confirmed in patient 8 (table 2). Two newborns (9 and 10) had episodes of PJRT, one on Holter monitoring in the immediate postnatal period and the other at two weeks of age; a third patient (case 12) had an AET episode. These three neonates were treated successfully with sotalol. The last patient (case 11) with tuberous sclerosis and cardiac tumours had frequent premature atrial contractions and runs of AET. He did not respond to digoxin, sotalol, and flecainide. A permanent pacemaker was implanted following episodes of sinus pauses ( $>5$ seconds) after bouts of tachycardia.

\section{Intra-atrial re-entrant tachycardia (flutter)}

Prenatal data

Six fetuses had sustained tachycardia caused by intra-atrial re-entry (table 1 ). They were in atrial flutter predominantly with 2:1 block but with occasional 1:1 or 3:1 AV conduction (fig 7). According to our protocol, all six fetuses were administered digoxin and four of them $(14,16,17,18)$ converted to sinus rhythm in utero. Fetus 13 was first seen at 36 weeks in severe hydrops. After a loading dose of digoxin, caesarean section was performed. Emergency caesarean section also had to be performed for fetus 15 one week after the initiation of treatment since the flutter was still present and the fetus's condition was deteriorating.

\section{Postnatal data}

The four fetuses who converted in utero remained in sinus rhythm after birth (table 2). Fetus 15, who was delivered in severe cardiac failure and sustained tachycardia, died one day after birth. Brief periods of sinus rhythm allowed the identification of ventricular pre-excitation in this case.

\section{DISCUSSION}

The precise identification of the mechanism underlying a tachyarrhythmia is important since the pharmacological approach can be quite different. Another aspect to be considered in fetal treatment is that the medication has to be given to the mother, who then shares the potential drug related morbidities with her fetus. Therefore, when one contemplates treating a fetus with arrhythmia, it is of utmost importance, firstly, to establish the electrophysiological mechanism at the time of diagnosis; secondly, to give the most efficient drug at the lowest possible dose; and thirdly, to avoid as much as possible blind trials of multiple drugs that increase the risk of maternal and fetal morbidity. It is noteworthy that in the present study, the need for postnatal treatment was infrequent (8 of 18 fetuses). This in all likelihood is related to maturational changes known to occur in the electrophysiology of the fetal heart.

In the presence of short VA re-entrant tachycardia, the pattern observed on the SVC/AA Doppler tracings was striking, characterised by a tall retrograde A wave superimposed on the aortic ejection wave. This typical pattern in re-entry tachycardia through a fast conducting retrograde limb is caused by the occurrence of atrial contractions while the AV valves are still closed. As expected, this cannon wave disappears or reappears suddenly with the conversion or reappearance of the tachycardia (fig 3). These observations lead to two important comments. Firstly, assessment of fetal ventricular diastolic function based on venous Doppler velocimetry is impossible during short VA tachycardia; the frequent interpretation of these tall A waves as being a sign of secondary cardiomyopathy must therefore be avoided. Secondly, in the presence of incessant tachyarrhythmia, the normal appearance of the venous A wave favours long VA tachycardia.

All drugs that can increase the refractory period of one limb of the circuit can be expected to be effective against the majority of re-entrant tachycardia found in utero. In our protocol, digoxin was chosen because of the vast experience gained with this agent in prenatal antiarrhythmic treatment. The five fetuses with typical re-entrant SVT (short VA) responded to digoxin. The SVC/AA approach helped to diagnose two other cases with short VA tachycardia but no re-entry, one with a junctional ectopic tachycardia and the other with an AET but first or second degree AV block. Neither fetus responded to digoxin alone. These cases are interesting inasmuch as they illustrate that a minority of fetuses with short VA SVT do not suffer from a re-entrant phenomenon.

For long VA tachycardia, our choice was to give a type III antiarrhythmic agent. We elected to give sotalol instead of amiodarone because of its good transplacental transfer, ${ }^{4}$ its relatively short half life, and its weak $\beta$ blocker effect. Its electrophysiological actions on the immature mammalian heart have been studied. ${ }^{5}$ Furthermore, favourable experiences with the use of sotalol in the paediatric age group have been documented. ${ }^{6-8}$ A case of sotalol induced torsades de pointes tachycardia in a 15 month old child has, however, been reported. ${ }^{9}$ By comparison, amiodarone has a long half life (between 3-6 weeks) and carries the risk of thyroid function impairment, which, because of growth and maturational processes occurring in the fetus and during the perinatal period, must be considered to be a major complication. Pulmonary fibrosis is another side effect that has been reported in an infant treated with amiodarone for SVT. ${ }^{10}$ The fact that one of the four fetuses with a prenatal diagnosis of AET turned out to have a PJRT in the postnatal investigation stresses the difficulty of differentiating AET from PJRT before birth. The only clue to the slow re-entrant tachycardia that characterises PJRT is the evidence of sudden arrest and onset of arrhythmia triggered by extrasystoles (fig 6). Fortunately, sotalol is effective for both AET and PJRT.

Our constant concern for maternal wellbeing explains our policy of using only one drug in the presence of flutter. In a previous study, we found that this approach could be rewarding and the use of another medication was frequently not necessary. ${ }^{11}$ The only death was, however, in this flutter group. The fetus was already in a state of advanced hydrops with 
severe cardiomyopathy. The association of intra-atrial reentrant tachycardia with AV accessory pathways, as observed in this last case, has been underlined previously. ${ }^{12}$

Despite the relatively small number of cases, the present report is encouraging inasmuch as it shows that precise identification of the mechanism underlying fetal tachyarrhythmia is possible in utero with the SVC/AA Doppler approach. In this respect, SVC/AA recordings give information similar to that obtained after birth with transoesophageal ECG. This investigation confirms that the mechanism of fetal SVT can be quite varied. There are therefore no grounds for using a common antiarrhythmic agent as a drug of first choice for all cases of fetal SVT, as currently advocated. The medication used in this study remain under investigation and is not claimed to be an ideal protocol. Previous experience and confidence with a specific drug is still a significant influential factor in the choice of an antiarrhythmic agent. Nevertheless, the management proposed here has, so far, yielded good results and no major modification appears to be justified for the moment.

\section{Authors' affiliations}

J-C Fouron, F Proulx, J L Bigras, C Boutin, M Brassard, S Gamache, Fetal Cardiology Unit, Department of Pediatrics, Sainte-Justine Hospital, University of Montreal, Montreal, Quebec, Canada

A Fournier, J Lamarche, Cardiology Division, Department of Pediatrics, Sainte-Justine Hospital

\section{REFERENCES}

1 Benditt DG, Pritchett ELC, Smith WM, et al. Ventriculoatrial intervals: diagnostic use in paroxysmal supraventricular tachycardia. Ann Intern Med 1979:91:161-6.

2 Jaeggi E, Fouron JC, Fournier A, et al. Ventriculo-atrial time interval measured on $M$-mode echocardiography: a determining element in diagnosis, treatment, and prognosis of fetal supraventricular tachycardia. Heart 1998:79:582-7.

3 Fouron JC, Proulx F, Miró J, et al. Doppler and M-mode ultrasonography to time fetal atrial and ventricular contractions. Obstet Gynecol 2000;96:732-6.

4 Erkkola R, Lammintausta R, Liukko P, et al. Transfer of propanolol and sotalol across the human placenta: their effect on maternal and fetal plasma renin activity. Acta Obstet Gynecol Scand 1982:61:31-4.

5 Houyel L, Fournier A, Ducharme G, et al. Electrophysiologic effects of sotalol on the immature mammalian heart. J Cardiovasc Pharmacol 1992;19:134-9.

6 Tipple M, Sandor G. Efficacy and safety of oral sotalol in early infancy. Pacing Clin Electrophysiol 1991;14:2062-5.

7 Maragnès $\mathbf{P}$, Tipple $M$, Fournier A. Effectiveness of oral sotalol for treatment of pediatric arrhythmias. Am J Cardiol 1992;69:751-4.

8 Tanel RE, Walsh EP, Lulu JA, et al. Sotalol for refractory arrhythmias in pediatric and young patients: initial efficacy and long-term outcome. Am Heart J 1995:130:791-7.

9 Sasse M, Paul T, Bergmann P, et al. Sotalol associated torsades de pointes tachycardia in a 15-month-old: successful therapy with magnesium aspartate. Pacing Clin Eletrophysiol 1998;21:1164-6.

10 Bowers PH, Fields J, Schwatz D, et al. Amiodarone induced pulmonary fibrosis in infancy. Pacing Clin Electrophysiol 1998;21:1665-7.

11 Jaeggi E, Fouron JC, Drblik SP. Fetal atrial flutter: diagnosis, clinical features, treatment and outcome. J Pediatr 1998;132:335-9.

12 Naheed ZJ, Strasburger JF, Deal BJ, et al. Fetal tachycardia: mechanisms and predictors of hydrops fetalis. J Am Coll Cardio 1996;27:1736-40.

\section{IMAGES IN CARDIOLOGY}

\section{Himalayan $\mathrm{P}$ waves in a patient with combined tricuspid and pulmonic stenosis}

$\mathrm{A}$ n 8 year old girl with a history of a murmur since early childhood had progressive shortness of breath and palpitations for two years. Two dimensional echocardiogram showed thickening of the tricuspid valve and pulmonic valve with a notably enlarged right atrium. A colour flow, Doppler examination revealed severe tricuspid valve stenosis with severe regurgitation and severe pulmonic stenosis. The findings of echocardiography were confirmed by catheterisation. Carcinoid syndrome, Leopard syndrome, and Noonan's syndrome were ruled out. The patient was diagnosed as having congenital combined tricuspid and pulmonic stenosis and referred directly for cardiac surgery.

Her recent 12 lead ECG revealed important diagnostic information and signs of right ventricular and right atrial pressure overloading consistent with pulmonary valve and tricuspid valve severe stenosis. It shows a normal sinus rhythm, right atrial enlargement, and

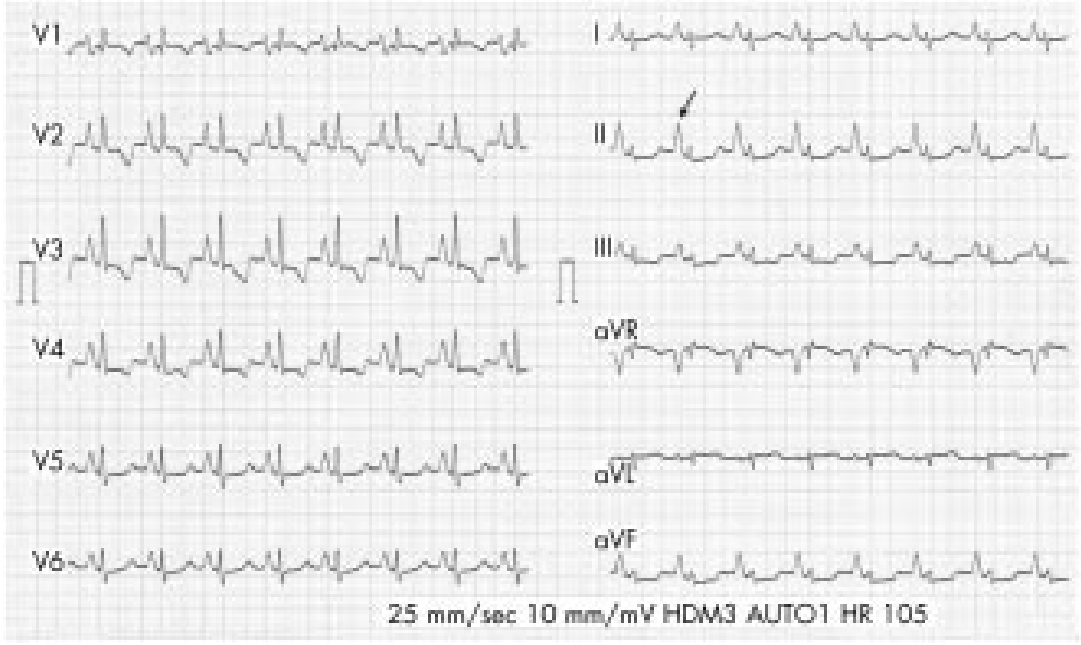

right ventricular hypertrophy with right ventricular forces. The $\mathrm{P}$ waves are tall $(>5 \mathrm{~mm}$ ) and peaked in lead II (black arrow). These types of $\mathrm{P}$ waves are called giant $\mathrm{P}$ waves or Himalayan $\mathrm{P}$ waves and are caused by reflected dilated right atrium resulting from pressure overloading.

V Davutoglu

M Kilinc

M H Dinckal

vedatdavutoglu@hotmail.com 